\title{
APPLICATION OF THE BISMUTH FILM ELECTRODE FOR THE DETERMINATION OF SELENIUM IN VITAMINIC COMPLEX BY SQUARE WAVE VOLTAMMETRY
}

\author{
BIANCHIN, Joyce Nunes ${ }^{1}$; DOS SANTOS, Marcel Silveira²; SPINELLI, Almir ${ }^{3}$; \\ 1,2,3 Universidade Federal de Santa Catarina, Departamento de Química, campus universitário, CEP 88040900 , \\ Florianópolis - SC, Brasil \\ * e-mail: jocca_bianchin@yahoo.com.br
}

Received 13 August 2009; received in revised form 12 October 2009; accepted 30 October 2009

\section{RESUMO}

Neste estudo foi desenvolvida uma metodologia analítica para a determinação de selênio em amostra de complexo vitamínico utilizando a voltametria de onda quadrada (VOQ). As análises foram realizadas com um eletrodo de cobre modificado com filme de bismuto (BiFE) depositado in situ pela aplicação de um potencial de $-400 \mathrm{mV}$ por 60 segundos numa solução contendo $\mathrm{HCl} 0,2 \mathrm{~mol} \mathrm{~L}^{-1}, \mathrm{KI}^{2} 2 \mathrm{~g} \mathrm{~L}^{-1}$ e $500 \mathrm{~g} \mathrm{~L} \mathrm{~L}^{-1}$ de Bi (III). As variáveis - tempo e potencial de condicionamento, foram otimizadas por VOQ para a formação do filme de bismuto. Em seguida, as variáveis que afetam a sensibilidade analítica para a redução do Se (IV) - frequência, incremento e a altura de pulso, também foram otimizadas. Após a otimização, foram obtidos os parâmetros analíticos de mérito, a saber: faixa linear de trabalho $90-1180 \mathrm{~g} \mathrm{~L} \mathrm{~L}^{-1}$; coeficiente de correlação $(R)=0,9998$. A metodologia mostrou excelente precisão, calculada como o desvio padrão relativo $(\operatorname{RSD} \%)=3,52 \%(n=5$, $150 \mu \mathrm{g} \mathrm{L}^{-1}$ ) ; limite de detecção de $26,4 \mu \mathrm{g} \mathrm{L}^{-1}$; limite de quantificação de $87,9 \mu \mathrm{g} \mathrm{\textrm {L } ^ { - 1 }}$. Frequência analítica de 30 amostras/hora. A amostra do complexo vitamínico foi analisada e apresentou concentração de selênio de 24 $\mu \mathrm{g} /$ comprimido. Por fim, um estudo de recuperação foi realizado obtendo-se $92 \%$.

Palavras-chave: Selênio, polivitamínico, voltametria de onda quadrada (VOQ), eletrodo de filme de bismuto (BiFE).

\begin{abstract}
In this work was developed an analytical methodology for determination of selenium in samples of vitaminic complex using the square wave voltammetry (SWV). The analysis were performed with a copper electrode modified with bismuth film deposited in situ by applying a potential of $-400 \mathrm{mV}$ for 60 seconds in a solution containing $\mathrm{HCl} 0.2 \mathrm{~mol} \mathrm{~L}^{-1}, 20 \mathrm{~g} \mathrm{~L}^{-1}$ and $500 \mathrm{\mu g} \mathrm{L}^{-1}$ of Bi(III). The variables: time and conditioning potential were optimized by SWV for the formation of the bismuth film, after that, the variables that affect the analytical sensitivity for the reduction of Se (IV): frequency, increment and high of pulse were also optimized. The linearity of the method ranged from $90-1180 \mu \mathrm{g} \mathrm{L}^{-1}$. Limit of detection of $26,4 \mu \mathrm{g} \mathrm{L}^{-1}$, quantification limit of $87,9 \mathrm{~g} \mathrm{~L} \mathrm{~L}^{-1}$. Analytical frequency of 30 samples/hour. The method showed excellent precision, calculated as the relative standard deviation (RSD\%) $(n=5)$ using spiked solution of $150 \mu \mathrm{g} \mathrm{L}^{-1}$. The concentration of selenium found in the sample of the vitaminic complex was $24 \mu \mathrm{g} /$ tablet. A study of recovery was performed resulting in $92 \%$.
\end{abstract}

keywords: Selenium, polivitaminic, square wave voltammetry, bismuth film electrode (BiFE) 
Introdução

Selênio é um elemento que pode ser considerado essencial ou tóxico para homens e animais dependendo da concentração na qual é administrado (ALEIXO \& et al., 2000). Ele está presente em ambientes aquáticos, sistemas biológicos e até mesmo na atmosfera (LONG \& NAGAOSA, 2007), e a principal fonte para o ser humano se constitui no seu uso em dietas alimentares. (por exemplo, nos complexos vitamínicos) (VENTURA \& et al., 2009). Segundo a Recommended Dietary Allowance (RDA) o selênio deve ser ingerido em até $70 \mu \mathrm{g} / \mathrm{dia}$ (PANIGATIA \& et al., 2007). Este metal pode ser encontrado tanto na forma inorgânica quanto orgânica (REYES \& et al., 2009).

A função mais conhecida do selênio é a de antioxidante, levada a cabo por meio da associação desse elemento com a enzima glutationa peroxidase. Esta atua na manutenção de grupos sulfidrilas vitais na forma reduzida, na síntese de hormônios derivados do ácido araquidônico, no metabolismo de compostos estranhos ao organismo, entre outros (FERREIRA \& et al., 2002).

Uma aplicação importante, recém descoberta, segundo Associação dos Laboratórios Farmacêuticos Nacionais, é que ele pode ser utilizado em um novo tratamento a fim de tratar complicações cardíacas decorrentes da doença de Chagas. O tratamento está sendo desenvolvido no Rio de Janeiro, nos laboratórios do Instituto Oswaldo Cruz (IOC). Trata-se de uma iniciativa pioneira uma vez que não existe nenhum medicamento para combater as complicações cardíacas desta doença descoberta no início do século $\mathrm{XX}$, por Carlos Chagas, e que atinge 3 milhões de brasileiros. $O$ medicamento à base de selênio funciona como um antioxidante, ou seja, restaura as células cardíacas e as preserva da oxidação que ocorre com a inflamação provocada pela doença. Por outro lado, a intoxicação por selênio provoca inúmeras reações, como o gosto metálico na boca, unhas e cabelos frágeis, irritação das vias respiratórias e perturbação das enzimas hepáticas, sendo que a intoxicação aguda pode provocar convulsões. Há grande ocorrência de intoxicações crônicas em populações indígenas de regiões cujo solo é muito rico em selênio e em pessoas expostas profissionalmente ao produto como ocorre nas indústrias químicas de semicondutores e de produtos cosméticos e dermatológicos (RIBEIRO \& et al., 2005).
Inúmeras técnicas têm sido desenvolvidas para a determinação de selênio em diversos tipos de amostras, sendo frequentemente empregadas espectrometria de absorção atômica, voltametria, espectrometria de absorção atômica por geração de hidreto, espectrometria de massa com plasma indutivamente acoplado, espectrometria de absorção atômica com atomizacão eletrotérmica, entre outras. (FOSTER \& SUMAR, 1996; ZHENGJUNA \& et al., 2005; NAKANO, YOSHII \& KAWASHIMA, 2004; BEDNAR, KIRGANA \& JONESA, 2009; GARCIA \& et al., 2005).

Os métodos voltamétricos, quando comparados com técnicas que envolvem espectroscopia atômica, podem oferecer algumas vantagens como: (1) instrumentação relativamente barata e fácil de manusear, (2) baixos limites de detecção e (3) determinação dada com uma boa precisão e exatidão (PANIGATI \& et al., 2007). A voltametria de onda quadrada (SWV), do inglês "Square Wave Voltammetry", é uma das técnicas voltamétricas de pulso mais rápidas e sensíveis. Essa técnica possui inúmeras vantagens, podendo-se destacar a ausência da interferência proveniente da corrente capacitiva, o que melhora a resolução dos dados experimentais, a sensibilidade analítica e, ainda, possibilitação de realizar análises rápidas (SOUZA \& et al., 2004). Os limites de detecção podem ser comparados aos das técnicas cromatográficas e espectroscópicas. Além disso, a análise dos parâmetros característicos desta técnica também possibilita a avaliação cinética e mecanística do processo eletródico em estudo (SOUZA, MACHADO \& AVACA, 2003).

Entre os eletrodos existentes, os mais utilizados em voltametria ainda são os de mercúrio (RAYMUNDO, PAULA \& FRANCO, 2007). Porém, devido à grande toxicidade destes, eletrodos modificados estão sendo propostos (HUTTON \& et al., 23006; TESAROVÁ \& et al., 2008; HUTTON, HOCEVER \& OGOREVC, 2005; DEMETRIADES, ECONOMOU \& VOULGAROPOULOS, 2004; KROLICKA, \& BOBROWSKI, 2004). O eletrodo de bismuto vem se destacando como material alternativo na construção de eletrodos por apresentar uma baixa toxicidade e possuir características similares ao eletrodo de mercúrio (WANG \& LU, 2000). Outras vantagens incluem a capacidade de formar ligas com metais pesados de forma análoga a amálgamas de mercúrio (ECONOMOU \& FIELDEN, 2003), 
permitindo a sua utilização em análises voltamétricas.

Neste estudo, desenvolveu-se uma metodologia analítica baseada em eletrodo de cobre modificado com filme de bismuto para a determinação de selênio em complexos vitamínicos, no intuito de monitorar a concentração desse metal em vitaminas para que esse elemento seja consumido de forma adequada pela população.

\section{Parte Experimental}

\section{Instrumentação}

As determinações voltamétricas foram realizadas em um potenciostato/galvanostato EG\&G PARTM, modelo 263A, em conjunto com o software M270. As medidas eletroquímicas foram realizadas em temperatura ambiente em uma célula de vidro com capacidade para $25 \mathrm{~mL}$, confeccionada em vidro Pyrex, acoplada a um sistema de três eletrodos.

O filme de bismuto foi depositado sobre o eletrodo de cobre, o qual foi usado como eletrodo de trabalho. O eletrodo de cobre foi previamente polido com uma pasta de alumina $0,05 \mu \mathrm{m}$ e logo rinsado com água deionizada. Como eletrodo de referência, utilizou-se $\mathrm{Ag} / \mathrm{AgCl}$ e um eletrodo de platina como eletrodo auxiliar.

\section{Reagentes e Soluções}

Todas as soluções foram preparadas empregando-se água deionizada proveniente de um sistema de purificação de água Milli- $Q{ }^{\circledR}$ da Millipore $^{\circledR}$ (Bedford, MA, USA). Todos os reagentes usados foram de grau analítico. Toda a vidraria de laboratório foi lavada com detergente neutro, mantida durante uma noite em solução de ácido nítrico $10 \%(\mathrm{v} / \mathrm{v})$ e a seguir enxaguada com água deionizada.

Soluções de trabalho foram preparadas diariamente, a partir de diluições adequadas de uma solução estoque (grau absorção atômica) de Se (IV) $1042 \mathrm{mg} \mathrm{L}^{-1}$. Soluções de Ácido clorídrico (Nuclear, Diadema, SP), iodeto de potássio (Nuclear), nitrato de bismuto (Sigma-Aldrich Milwaukee, USA), foram preparadas e estocadas.

\section{Procedimento Analítico}

A deposição do filme de bismuto sobre o eletrodo de cobre foi realizada in situ, em solução de $\mathrm{HCl} 0,2 \mathrm{~mol} \mathrm{~L}^{-1}$, KI $20 \mathrm{~g} \mathrm{~L}^{-1}$ e $\mathrm{Bi}(\mathrm{III}) 500 \mu \mathrm{g} \mathrm{L}^{-1}$, pela aplicação de um potencial de $-400 \mathrm{mV}$ sob agitação constante durante 60 segundos. Os voltamogramas foram obtidos através da varredura de potencial de $-400 \mathrm{mV}$ a $-1400 \mathrm{mV}$, através da técnica de VOQ. A limpeza do eletrodo foi realizada manualmente com alumina $0,05 \mu \mathrm{m}$ e, em seguida, procedeu-se à agitação ultrassônica durante 30 segundos.

A espécie de selênio predominante em $\mathrm{HCl} 0,2 \mathrm{~mol} \mathrm{~L}^{-1}$ é $\mathrm{H}_{2} \mathrm{SeO}_{3}$. Em um potencial de deposição $-500 \mathrm{mV}$, o Se (IV) é reduzido para $\mathrm{H}_{2} \mathrm{Se}$ como resultado de um processo de transferência de $6 \mathrm{e}^{-}$juntamente com a formação do filme de bismuto, e por fim $\circ \mathrm{H}_{2} \mathrm{Se}$ reduzido é adsorvido sobre o eletrodo.

\section{Resultados e Discussão}

\section{Otimização dos parâmetros de preparação do BiFE}

Testes preliminares, de acordo com Long e colaboradores (LONG, NAGAOSA \& Yukio, 2007) utilizando solução de $500 \mu \mathrm{g} \mathrm{L}^{-1}$ de $\mathrm{Bi}(\mathrm{III})$,

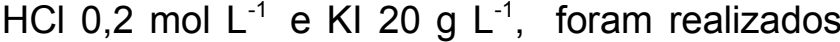
para verificar a formação e o comportamento do filme de bismuto na superfície do eletrodo de cobre. O filme se mostrou uniforme e o sinal foi reprodutível.

Após fixar a concentração de bismuto e

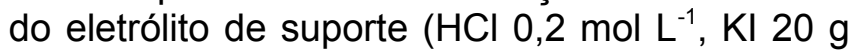
$\mathrm{L}^{-1}$ ), uma otimização do potencial e tempo de deposição foi realizada. O efeito do tempo de deposição do BiFE foi avaliado utilizando a voltametria de onda quadrada. A concentração de selênio foi de $5 \times 10^{-5} \mathrm{~mol} \mathrm{~L}^{-1}$ e o potencial de deposição utilizado foi de $-400 \mathrm{mV}$.

Conforme a Figura 1, tempo de deposição versus densidade de corrente, pode-se observar que a intensidade dos picos aumenta linearmente com 0 aumento do tempo de deposição até 50 segundos. A partir de 60 segundos, nota-se a perda da linearidade. Logo, um tempo maior que 60 segundos, não leva a um ganho de resposta analítica. O tempo de 60 segundos foi escolhido por estar no início da faixa em que o gráfico assume comportamento constante.

O potencial de deposição para a formação do filme foi estudado de $-300 \mathrm{mV}$ até $-700 \mathrm{mV}$. Observa-se na Figura 2 que a aplicação de potenciais mais negativos, de -500 a $-700 \mathrm{mV}$, não proporcionaram uma boa sensibilidade, apesar da formação do filme de $\mathrm{Bi}(\mathrm{II})$ ter ocorrido. Em $-300 \mathrm{mV}$, nota-se uma queda na densidade de corrente. Isso se deve ao fato de que, próximo a este potencial, inicia-se a oxidação do bismuto e consequentemente a 
diminuição do pico. Por fim, o potencial de -400 $\mathrm{mV}$ foi escolhido para a deposição do bismuto sobre o eletrodo de cobre.

\section{Otimização dos parâmetros da voltametria de onda quadrada}

Um passo importante no desenvolvimento de uma metodologia eletroanalítica é a otimização dos parâmetros que podem influenciar a resposta voltamétrica. $\mathrm{Na}$ voltametria de onda quadrada, os principais parâmetros que podem influenciar na resposta analítica são: frequência $(f)$, altura de pulso $(\Delta \mathrm{H}$; $a=\Delta \mathrm{H} / 2$ ) e $\mathrm{o}$ incremento de varredura de potencial $(\Delta \mathrm{Es})$. Neste trabalho, o estudo dessas variáveis foi realizado em uma solução de $500 \mu \mathrm{g}$

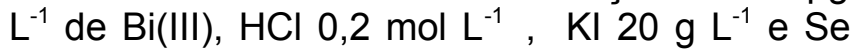
(IV) $5 \times 10^{-5} \mathrm{~mol} \mathrm{~L}^{-1}$, durante todas as análises.

A frequência é um dos parâmetros mais importantes da VOQ, pois determina a intensidade dos sinais, 0 que, consequentemente, reflete diretamente na sensibilidade do método (CABRAL, SOUZA \& MACHADO, 2003). O aumento da frequência ocasiona um aumento na corrente, o que resulta em uma melhor sensibilidade.

Neste estudo, variou-se a frequência de 5 a $35 \mathrm{~Hz}$. Segundo a Figura 3, pode-se observar que há um aumento da densidade de corrente com o aumento da frequência, de 5 até $15 \mathrm{~Hz}$. A partir de $20 \mathrm{~Hz}$ o gráfico perde linearidade, fica constante e logo em $35 \mathrm{~Hz}$ ocorre uma queda significativa do sinal analítico. Isso ocorre, tendo em vista que em frequências muito altas não se consegue separar a corrente faradaica da corrente capacitiva, ocorrendo então uma queda na resposta. Considerando erros experimentais, não há diferenças significativas na utilização de 15 a $30 \mathrm{~Hz}$. Como um compromisso ótimo entre o perfil voltamétrico e o sinal analítico, a frequência de $15 \mathrm{~Hz}$ foi selecionada.

Outro fator importante na VOQ é a altura do pulso, que, quando aumentada, aumenta-se o valor da corrente, proporcionando uma maior sensibilidade. Entretanto, um aumento excessivo provoca também um alargamento do pico, reduzindo a resolução do mesmo. O efeito da altura de pulso sobre a corrente de pico foi avaliada de 10 a $30 \mathrm{mV}$. Os resultados estão expostos na Figura 4. Observa-se que houve um aumento na densidade de corrente com o aumento da altura de pulso até $25 \mathrm{mV}$. Porém, em $30 \mathrm{mV}$, houve uma queda significativa no sinal analítico, e a resolução do gráfico foi afetada, sendo então adotada como altura de pulso de $25 \mathrm{mV}$ para demais análises.
O incremento de potencial é um dos responsáveis pela definição da velocidade de varredura de potenciais, já que esta é dada pelo produto do incremento de potencial pela frequência de aplicação dos pulsos de potencial.

$\mathrm{O}$ incremento foi estudado de 1 a $5 \mathrm{mV}$. $\mathrm{Na}$ Figura 5 está apresentada a influência da variação do incremento na resolução dos sinais analíticos. Observou-se que, com o aumento do incremento e, consequentemente, das velocidades de varreduras altas, ocorreu uma perda na resolução de pico. Sendo assim, a melhor resolução e sensibilidade foram verificadas com incremento de $4 \mathrm{mV}$.

Um estudo da natureza do processo eletródico sobre a superfície do eletrodo de cobre modificado com filme de bismuto foi realizado através da voltametria cíclica. A faixa de velocidades estudada foi de 20 a $100 \mathrm{mV} \mathrm{s}^{-1}$. Conforme mostra a Figura 6, o processo é irreversível (pois não apresenta pico de oxidação) e controlado por difusão. O gráfico de $\log \mathrm{i}$ em função de $\log v$ apresentou comportamento linear. Um comportamento linear com $\delta \log (i) / \delta \log (v)=0,5$ indica um processo de transporte de massa controlado por difusão e $\delta \log (i) / \delta \log (v)=1,0$ indica um processo controlado por adsorção (PEDROSA, CODOGNOTO \& AVACA, 2003). Considerando que $\delta \log (i) / \delta l o g(v)=0,54$, pode-se afirmar que o processo de transporte de massa é controlado difusionalmente. No mesmo experimento, um voltamograma de 10 ciclos na velocidade de 100 $\mathrm{mV} \mathrm{s}^{-1}$ foi construído a fim de verificar a ocorrência de adsorção do produto formado. A ausência do pico após os 10 ciclos, confirma que o produto é adsorvido no eletrodo de filme de bismuto.

Após as variáveis otimizadas, uma curva de calibração (Figura 7) com concentração de $50 \times 10^{-7} \mathrm{~mol} \mathrm{~L}^{-1}$ a $150 \times 10^{-7} \mathrm{~mol} \mathrm{~L}^{-1}$ de Se(IV) foi construída com o objetivo de obter as figuras analíticas de mérito, que estão representadas na Tabela 1.

A metodologia de análise pode ser avaliada pela aplicação de testes de recuperação, que consiste em adicionar à amostra uma determinada quantidade do analito. A concentração final deve ser igual à soma da concentração medida do analito na amostra e da concentração adicionada. Assim, o valor recuperado de analito, que foi adicionado, é a diferença entre o valor medido antes e após a adição sendo este representado na forma de percentual do valor adicionado, e é geralmente 
aceitável recuperação entre $80 \%$ e $120 \%$.

O sistema proposto foi utilizado para a determinação de Se (IV) em amostra de complexo vitamínico. Para verificar a exatidão do método, foram aplicados testes de recuperação, adicionando-se alíquotas para concentrações adicionadas de selênio, respectivamente, $50 \times 10^{-7}$ mol L-1, $100 \times 10^{-7} \mathrm{~mol} \mathrm{~L}^{-1}$ e $150 \times 10^{-7}$ na amostra. A amostra de vitamina analisada apresentou concentração de selênio de $24 \mu \mathrm{g} /$ comprimido, sendo o valor fornecido pelo fabricante de 25 $\mu \mathrm{g} /$ comprimido. Comparando-se a inclinação da reta com a inclinação da reta da curva de calibração, foi possível calcular a recuperação, a qual apresentou um resultado de $92 \%$, o que é bastante aceitável, levando em consideração a complexidade da matriz da amostra.

\section{Conclusão}

A aplicação do eletrodo de cobre modificado com filme de bismuto utilizando a voltametria de onda quadrada se mostrou simples e rápida e a formação do filme de bismuto apresentou ótima reprodutibilidade. Assim, a modificação de eletrodos com esse filme é mais uma alternativa muito atraente para a determinação não só de metais, mas também para compostos orgânicos. A metodologia proposta foi capaz de alcançar um limite de detecção em nível traço e com excelente precisão.

A amostra de vitamina analisada apresentou concentração de selênio muito próxima da concentração fornecida pelo fabricante. Esse tipo de monitoramento é de suma importância, pois a ingestão de doses excessivas de selênio pode provocar reações diversas para o ser humano.

\section{Referências}

1) ALEIXO, P. C.; NOBREGA, J. A.; SANTOS, Dário J.; MULLER, R. C. S. Determinação direta de selênio em água de coco e em leite de coco utilizando espectrometria de absorção atômica com atomização eletrotérmica em forno de grafite. Química Nova, V. 23, p. 310-312, 2000.

2) BEDNAR, A. J.; KIRGANA, R. A.; JONESA, W. T. Comparison of standard and reaction cell inductively coupled_plasma mass spectrometry in the determination of chromium and selenium species by HPLC-ICP-MS. Analytica Chimica Acta, V. 632, p. 27-34, 2009.

3) CABRAL, M. F.; SOUZA, D.; ALVES, C. R.; MACHADO, S. A. S. Square wave voltammetric study of the electrochemical behavior of the herbicide ametryne. Eclética Química, V. 28, p. 4147, 2003.

4) DEMETRIADES, D. ; ECONOMOU, A. ; VOULGAROPOULOS, A. A study of pencil-lead bismuth-film electrodes for the determination of trace metals by anodic stripping voltammetry. Analytica Chimica Acta, V. 519, p.167-172, 2004.

5) ECONOMOU, A.; FILDEN, P. R. Mercury film electrodes: developments, trends and potentialities for electroanalysis. Analyst, V. 128, p. 205-212, 2003.

6) FERREIRA, K. S.; GOMES, J. C.; BELLATO, C. R.; JORDAO, C. P. Selenium content of Brazilian foods. Revista Panamericana de Salud Publica, V. 11, p. 172-177, 2003.

7) FOSTER L. H.; SUMAR, S. Selenium concentrations in soya based milks and infant formulae available in the United Kingdom. Food Chemistry, V. 56, p. 93-98, 1996.

8) GARCIA, J. B.; KRACHLER, M.; CHEN, B.; SHOTYK, W. Improved determination of selenium in plant and peat samples using hydride generation-atomic fluorescence spectrometry (HG-AFS). Analytica Chimica Acta, V. 534, p. 255-261, 2005.

9) HUTTON, E. A.; HOCEVER, S. B.; MAUKO, L.; OGOREVC, B. Bismuth film electrode for anodic stripping voltammetric determination of tin. Analytica Chimica Acta, V. 580 , p. 244-250, 2006.

10) HUTTON, E. A.; HOCEVER, S. B.; OGOREVC, B. Ex situ preparation of bismuth film microelectrode for use in electrochemical stripping_microanalysis. Analytica Chimica Acta, V. 537, p. 285-292, 2005.

11) KRÓLICKA, A.; BOBROWSKI, A. Bismuth film electrode for adsorptive stripping voltammetry-electrochemical and 
microscopic study. Electrochemistry Communications, V. 6 p. 99-104, 2004.

12) LONG, J.; NAGAOSA, Y. Determination of Selenium(IV) by Catalytic Stripping Voltammetry with an in situ Plated Bismuth-film Electrode. Analytical Sciences, V. 23, p. 1343-1346, 2007.

13) NAKANO, S.; YOSHII, M.; KAWASHIMA, T. Flow-injection simultaneous determination of selenium(IV + VI) using photoxidative coupling of $p$ hydrazinobensenesulfonic acid with $\mathrm{N}$-(1naphthyl)ethylenediamine._Talanta, V. 64, p. 1266-1272, 2004.

14) PANIGATIA, M.; FALCIOLAB, L.; MUSSINIB, P.; BERETTAC, G.; FACINO, R. Determination of selenium in Italian rices by differential pulse cathodic stripping voltammetry. Food Chemistry, V. 105, p. 1091-109, 2007.

15) PEDROSA, V. D.; CODOGNOTO L.; AVACA, L. A. Electroanalytical determination of 4-nitrophenol by square wave voltammetry on diamond electrodes. Journal of the Brazilian Chemical Society, V. 14, p. 530-535, 2003.

16) RAYMUNDO, M.; PAULA, M.; FRANCO, C. V.; FETT, R. Quantitative determination of the phenolic antioxidants using voltammetric techniques. Food Science and Technology, V. 40, p. 1133-1139, 2007.

17) REYES L. H; MAR J. L; RAHMAN G. M; SEYBERT B.; FAHRENHOLZ T.; KINGSTON $H$. M. Simultaneous determination of arsenic and selenium species in fish tissues using microwaveassisted enzymatic extraction and ion chromatography-inductively coupled plasma mass spectrometry. Talanta, V.
78, p. 983-990, 2009.

18) RIBEIRO, L.; VIEIRA, E. M.; ALVES, M. G.; ALMEIDA, F. T. SBSR p. 3261-3268, 2005.

19) SOUZA, D.; CODOGNOTO, L.; MALAGUTTI, A. R.; TOLEDO, R. A.; PEDROSA, V. A.; OLIVEIRA, R. T. S. Voltametria de onda quadrada. Segunda parte: aplicações. Química Nova, V. 27, p. 790-797, 2004.

20) SOUZA, D.; MACHADO, S. A. S. and AVACA, Luis A. Voltametria de onda quadrada. Primeira parte: aspectos teóricos. Química Nova, V. 26, p. 81-89, 2003.

21) TESAROVÁ, E.; HERAS, A.; COLINA, A; RUIZ, V.; SVANCARA, I.; VYTRAS, K.; PALACIOS,J. L. A spectroelectrochemical approach to the electrodeposition of bismuth film electrodes and their use in stripping analysis. Analytica Chimica Acta, V. 608, p. 140-146, 2008.

22) VENTURA, M.; STIBILJ, V.; FREITAS, M. C.; PACHECO, A. Determination of ultratrace levels of selenium in fruit and vegetable samples grown and consumed in Portugal. Food Chemistry, V. 115, p. 200-206, 2009.

23) ZHENGJUNA, G.; XINSHENA, Z; GUOHEA, C.; ZINFENG, X. Flow injection kinetic spectrophotometric determination of trace amounts of $\mathrm{Se}$ (IV) in seawater. Talanta, V. 66, p. 1012-1017, 2005.

24) WANG, J.; LU, J. M. Bismuth film electrodes for adsorptive stripping voltammetry of trace nickel. Electrochemistry Communications, V. 6, p. 390-393, 2000. 


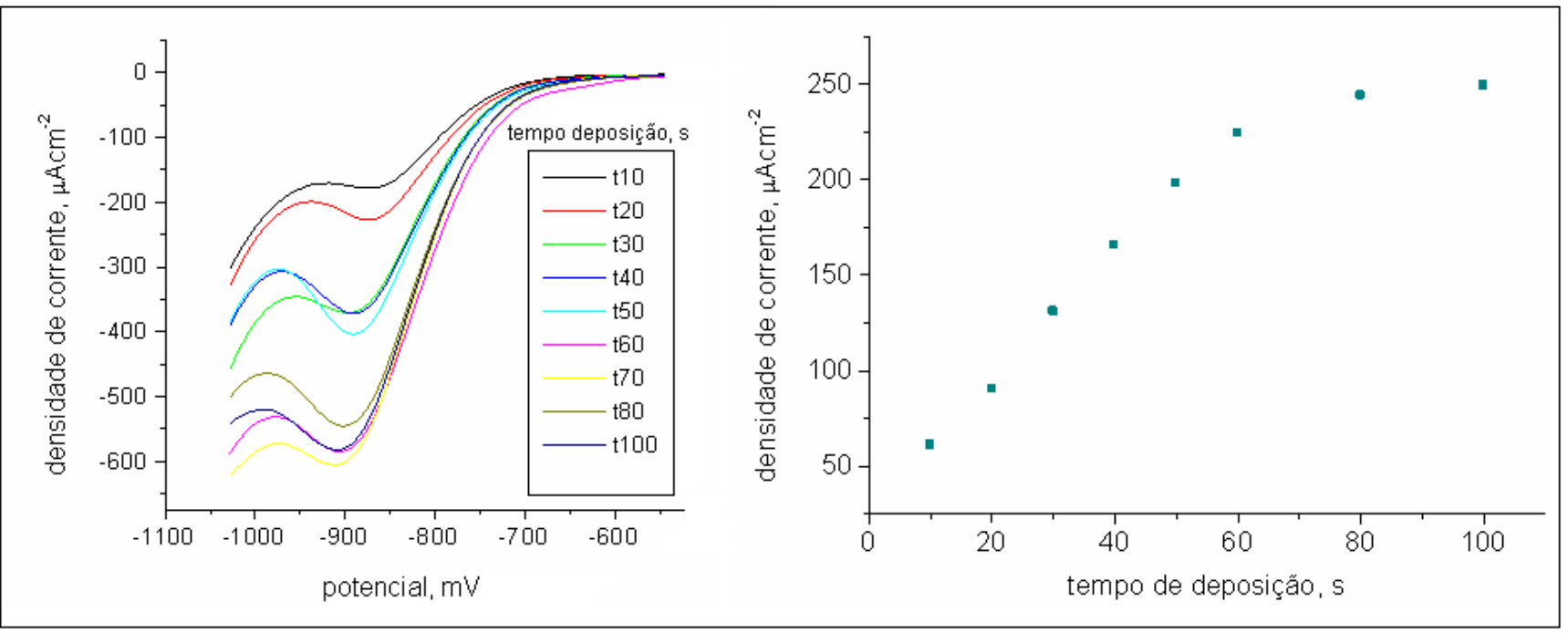

Figura 1- Influência do tempo de deposição do filme de bismuto sobre a densidade de corrente na determinação de selênio por voltametria de onda quadrada. (A) Voltamogramas para Se em diferentes tempos de deposição. (B) Linearidade da densidade de corrente em função do tempo de deposição. Concentração de Se $5 \times 10^{-5} \mathrm{~mol} \mathrm{~L}^{-1}$, concentração de $\mathrm{Bi}(\mathrm{III})$ de $500 \mu \mathrm{g} \mathrm{L}^{-1}, \mathrm{KI}_{20} \mathrm{~g} \mathrm{~L}^{-1}$ e de $\mathrm{HCl} 0,2 \mathrm{~mol} \mathrm{~L}^{-1}$. Potencial de deposição $-400 \mathrm{mV}$.

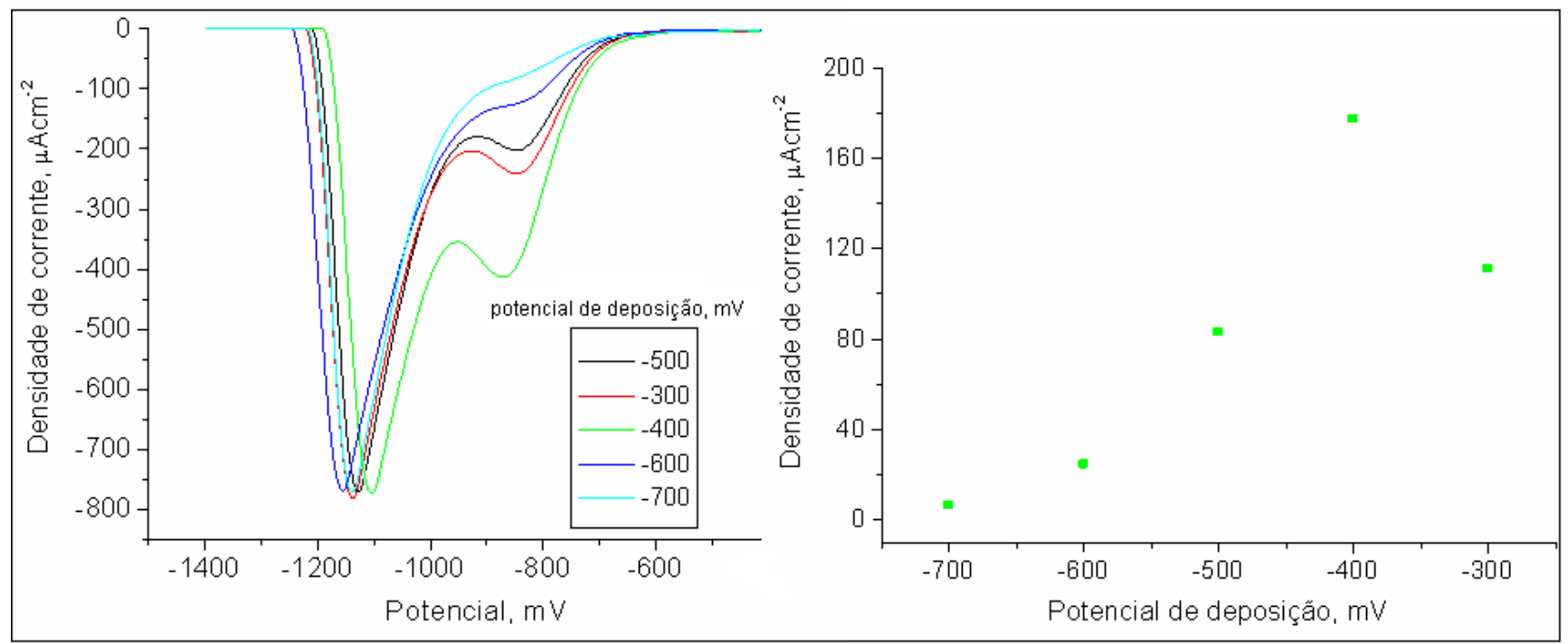

Figura 2- Influência do potencial de deposição sobre a densidade de corrente na determinação de selênio por voltametria de onda quadrada. (A) Voltamogramas para Se em diferentes potenciais de deposição. (B) Comportamento da densidade de corrente em função do potencial de deposição.

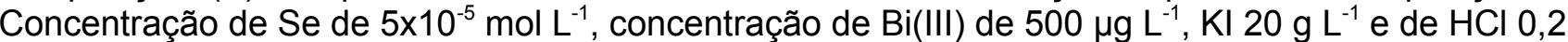
$\mathrm{mol} \mathrm{L}^{-1}$. Tempo de deposição de 60 segundos. 


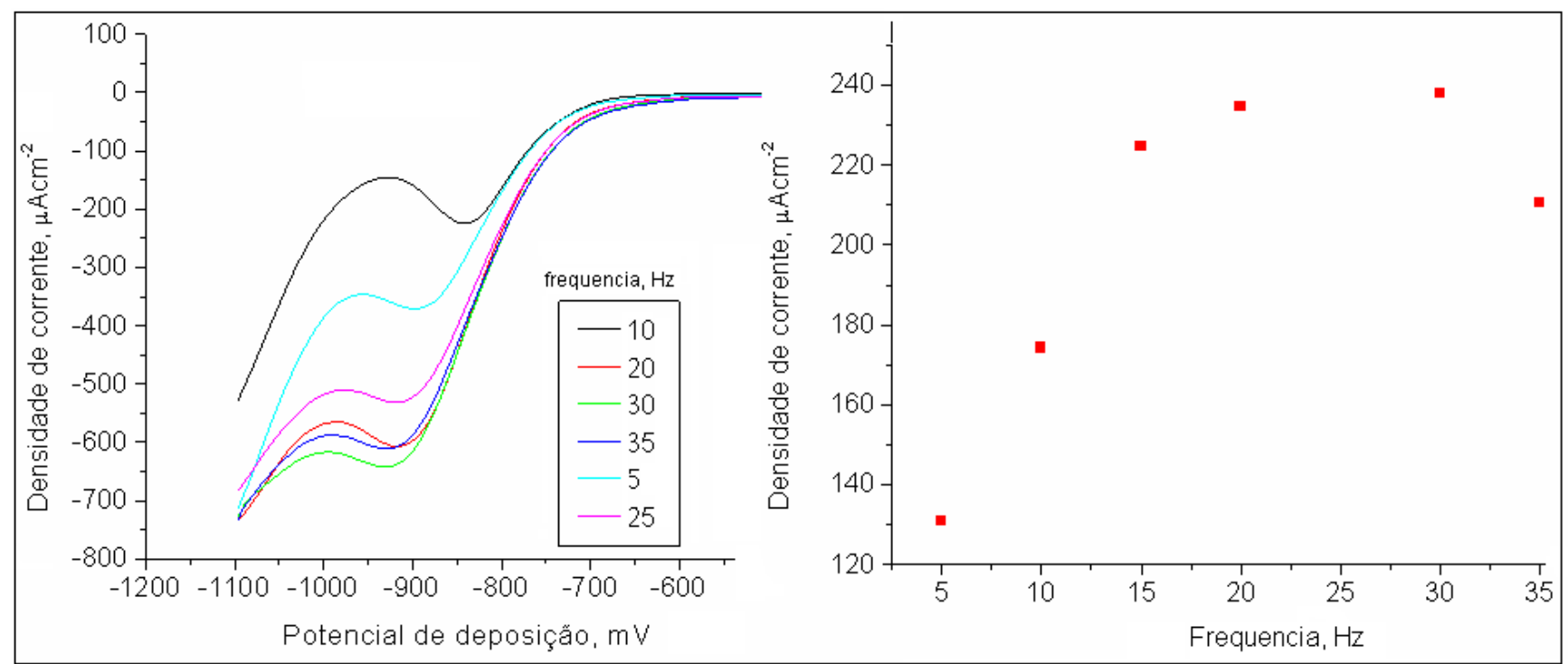

Figura 3- Influência da frequência sobre a densidade de corrente na determinação de selênio por voltametria de onda quadrada. (A) Voltamogramas para selênio em diferentes frequências. (B) Comportamento da densidade de corrente em função da frequência aplicada. Concentração de Se de $5 \times 10^{-5} \mathrm{~mol} \mathrm{~L}^{-1}$, concentração de $\mathrm{Bi}(\mathrm{III})$ de $500 \mathrm{\mu g} \mathrm{L}^{-1}, \mathrm{KI}_{20} \mathrm{~g} \mathrm{~L}^{-1}$ e de $\mathrm{HCl} \mathrm{0,2} \mathrm{mol} \mathrm{L}^{-1}$. Tempo de deposição de 60 segundos e potencial de deposição de $-400 \mathrm{mV}$.

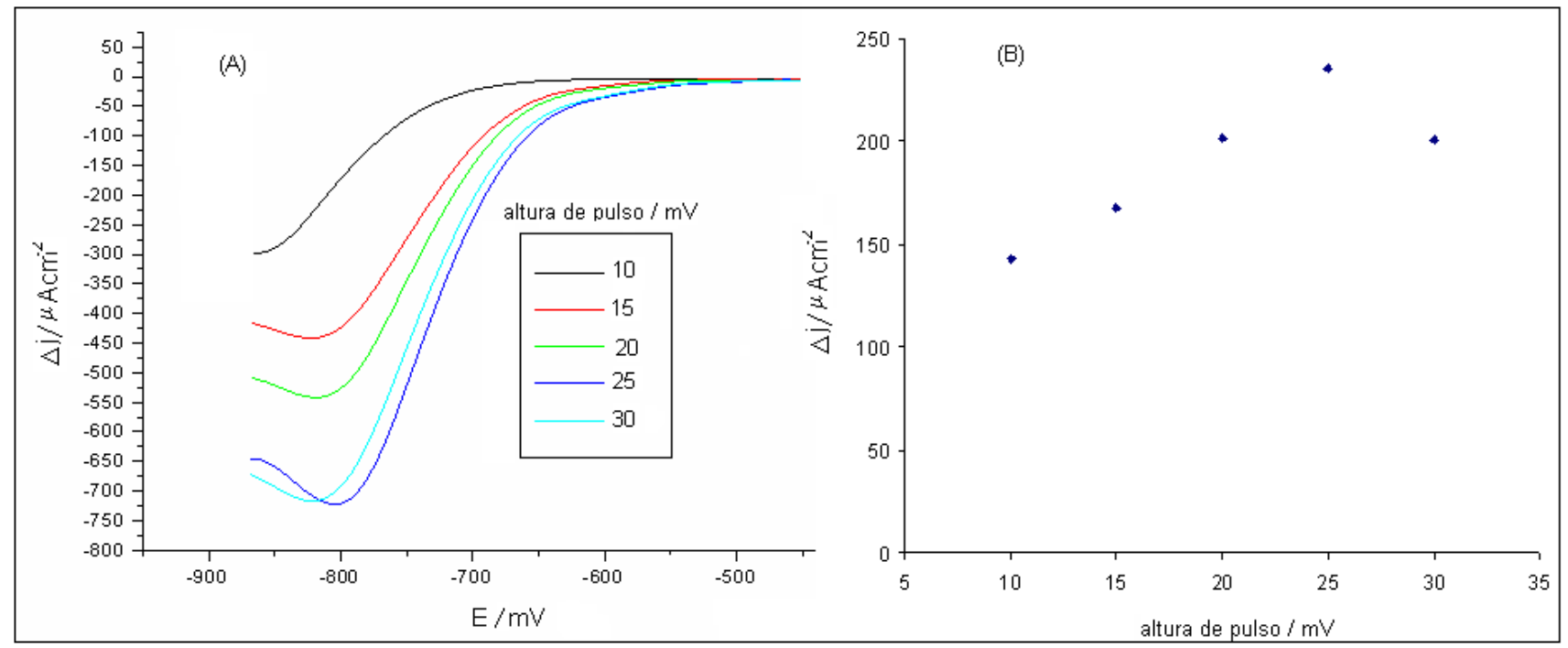

Figura 4- Influência da altura de pulso sobre a densidade de corrente para determinação de selênio por voltametria de onda quadrada. (A) Voltamogramas para Se em diferentes alturas de pulso. (B) Comportamento da densidade de corrente em função altura de pulso. Concentração de Se $5 \times 10^{-5} \mathrm{~mol}$

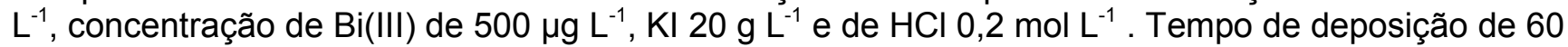
segundos e potencial de deposição de $-400 \mathrm{mV}$. 


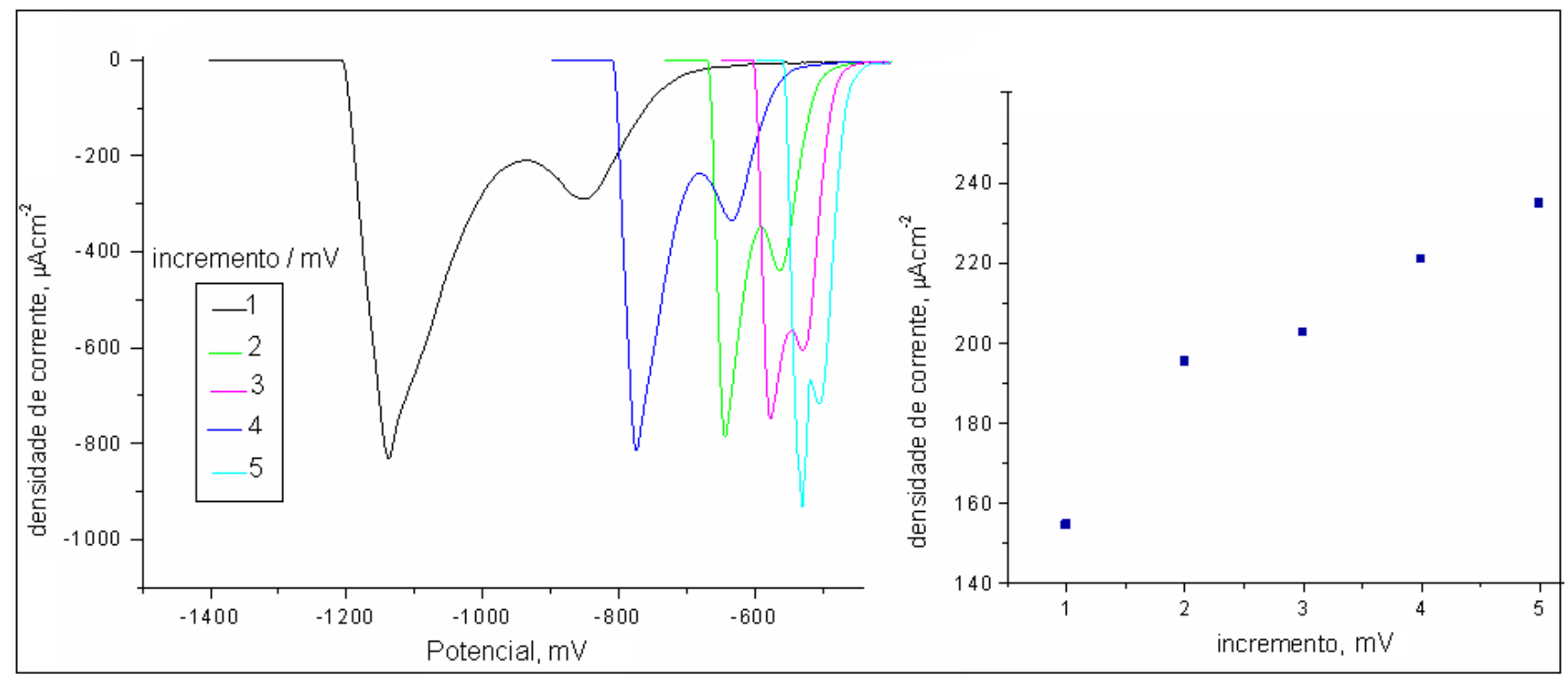

Figura 5- Influência do incremento de varredura de potencial sobre a densidade de corrente para determinação de selênio por voltametria de onda quadrada. (A) Voltamogramas para Se em diferentes incrementos. (B) Comportamento da densidade de corrente em função do incremento.

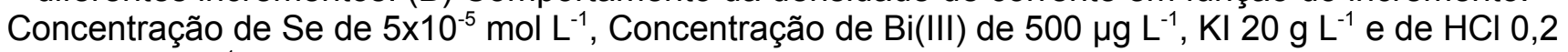
$\mathrm{mol} \mathrm{L}^{-1}$. Tempo de deposição de 60 segundos e potencial de deposição de $-400 \mathrm{mV}$.

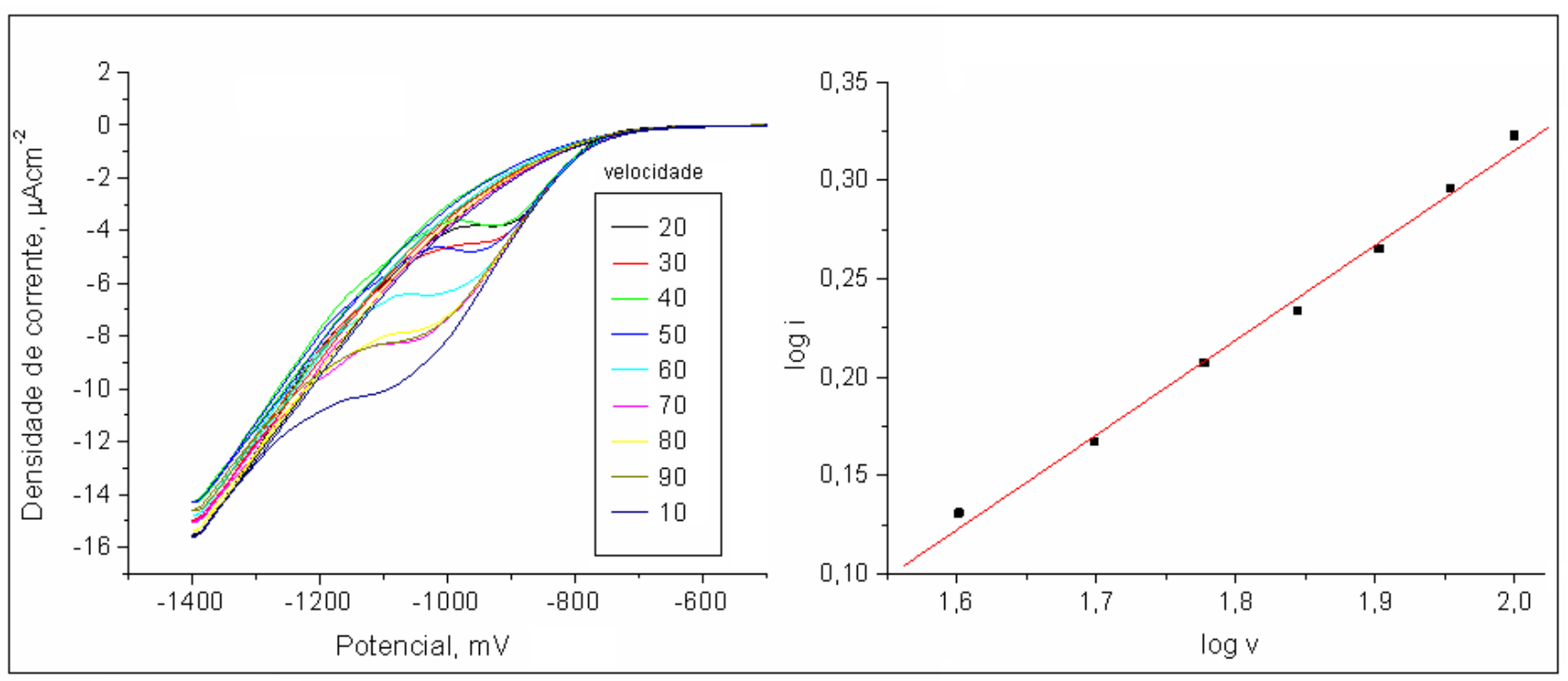

Figura 6- Influência da velocidade sobre a densidade de corrente na determinação de selênio por voltametria cíclica. (A) Voltamogramas em diferentes velocidades (B) Log i versus log $\mathrm{V}$ com slop 0,54 que caracteriza processo controlado por difusão. Concentração de Se de $5 \times 10^{-3} \mathrm{~mol} \mathrm{~L}^{-1}, \mathrm{Bi}(\mathrm{III})$ $500 \mu \mathrm{g} \mathrm{L}^{-1}, \mathrm{KI}^{2} 2 \mathrm{~g} \mathrm{~L}^{-1}$ e de $\mathrm{HCl} 0,2 \mathrm{~mol} \mathrm{~L}^{-1}$. Tempo de deposição de 60 segundos e potencial de deposição de $-400 \mathrm{mV}$. 


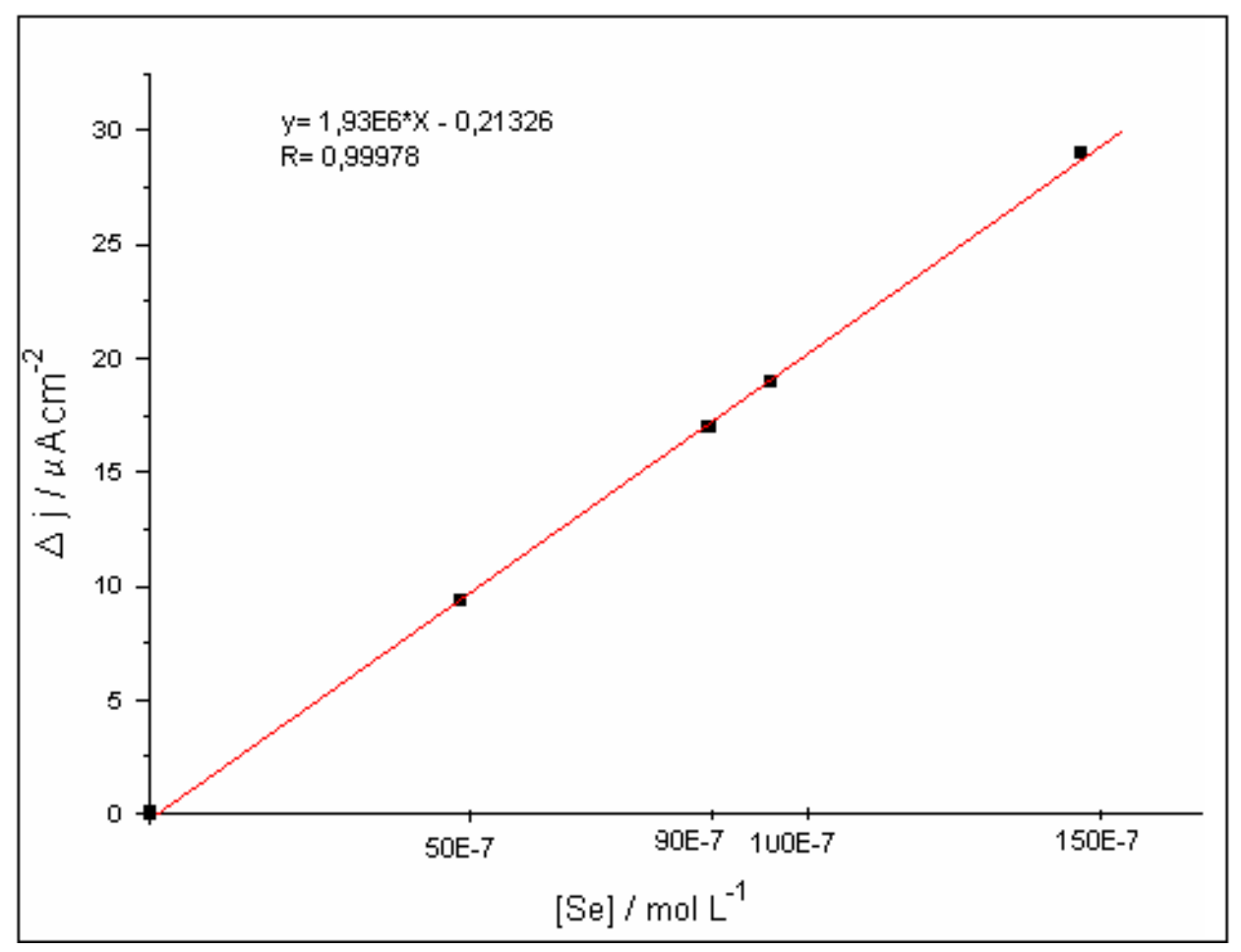

Figura 7- Curva de calibração para selênio usando eletrodo de filme de bismuto por voltametria de

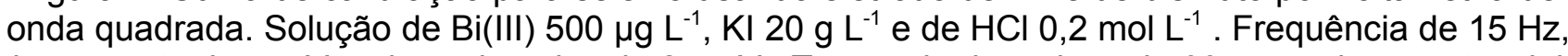
incremento de $4 \mathrm{mV}$ e altura de pulso de $25 \mathrm{mV}$. Tempo de deposição de 60 segundos e potencial de deposição de $-400 m V$.

Tabela 1- Parâmetros analíticos de mérito para determinação de selênio por voltametria de onda quadrada utilizando o filme de bismuto depositado sobre eletrodo de cobre.

\begin{tabular}{cc}
\hline Faixa linear de trabalho $\left(\mu \mathrm{g} \mathrm{L}^{-1}\right)$ & $85-1100$ \\
\hline Coeficiente de correlação linear $(\mathrm{R})$ & 0,9998 \\
\hline RSD \% $(n=5)$ & 2,52 \\
\hline Frequência analítica (amostras/hora) & 30 \\
\hline Limite de detecção $\left(\mu \mathrm{g} \mathrm{L}^{-1}\right)$ & $26,4 \mu \mathrm{L} \mathrm{L}^{-1}$ \\
\hline Limite de quantificação $\left(\mu \mathrm{g} \mathrm{L}^{-1}\right)$ & $87,9 \mu \mathrm{L} \mathrm{L}^{-1}$ \\
\hline
\end{tabular}

